\title{
Dust direct radiative effects on the earth-atmosphere system over east Asia: Early spring cooling and late spring warming
}

\author{
WANG Hong $^{1 *}$, ZHAO TianLiang ${ }^{2}$, ZHANG XiaoYe $^{1} \&$ GONG SunLing ${ }^{1,2}$ \\ ${ }^{1}$ Centre for Atmosphere Watch and Services, Chinese Academy of Meteorological Sciences, Beijing 100081, China; \\ ${ }^{2}$ Air Quality Research Division, Science \& Technology Branch, Environment Canada, Toronto, Ontario M3H 5T4, Canada
}

Received July 15, 2010; accepted December 14, 2010

\begin{abstract}
Focusing on three dust storms occurring in spring 2001, we developed a detailed aerosol parameterization scheme and integrated it in a radiative transfer model to characterize possible impacts of solar altitude angle on dust direct radiative effects over China desert regions and the North Pacific, using actual daily solar altitude angles. Increasing solar altitude angle from early spring (or winter) to late spring (or summer) leads to increase of positive clear sky radiative forcing, and decrease of negative radiative forcing due to dust aerosols at the top of the atmosphere. Because solar altitude angle increases from early to late spring, dust-clear sky radiative forcing may change from negative to positive at the top of atmosphere, showing a change from cooling to heating of the earth-atmosphere system over high-albedo deserts and nearby regions. Over low-albedo ocean negative clear sky radiative forcing by dust may decrease, suggesting a change from strong to weak cooling on the earth-atmosphere system. The impacts of solar altitude angle on cloudy sky radiative forcing due to dust are similar to those of clear sky. Impacts of low cloud on dust radiative forcing are the same as increasing surface albedo. This causes the transition of dust cooling effects into heating effects over deserts to occur earlier, and causes decrease of negative radiative forcing over the ocean and even cause a change from weak negative radiative forcing to weak positive forcing over local areas. Even in the same East Asian desert regions and nearby areas, the strength and sign of the radiative forcings depend on storm dates and thus solar altitude angle. The nearer to early spring (or winter) a dust storm occurs, the easier it leads to negative radiative forcing at the top of atmosphere, which indicates cooling effects on the earth-atmosphere system. In contrast, the nearer to late spring (or summer) a dust storm occurs, the easier it leads to positive radiative forcing at the top of atmosphere, showing heating effects. Over East Asian deserts and nearby regions, dust layers may be regarded as cooling sources in early spring (winter) and warming sources in late spring (summer).
\end{abstract}

solar altitude angle, radiation transfer model, radiative forcing, dust storm, radiative effects, aerosol parameterization scheme

Citation: Wang H, Zhao T L, Zhang X Y, et al. Dust direct radiative effects on the earth-atmosphere system over east Asia: Early spring cooling and late spring warming. Chinese Sci Bull, 2011, 56: 1020-1030, doi: 10.1007/s11434-011-4405-3

Solar altitude angle changes not only throughout a single day, but also seasonally. Aerosol radiative effects are very sensitive to the changes in solar radiation angle which influences the strength and sign of aerosol radiative forcing at the top of the atmosphere. The impacts of aerosol concentration, surface albedo and clouds on aerosol radiative forcing at the top of the atmosphere, and on radiative heating

*Corresponding author (email: wangh@cams.cma.gov.cn) rates, depend largely on the solar altitude angle [1-3]. Solar radiation reaching the ground is expressed generally as: $S_{1}=S \times \sin \zeta\left(\right.$ or $\left.S_{1}=S \times \cos \theta, \theta+\zeta=90\right)$, where $S$ is integrated solar radiant flux density at the surface vertical to the direction of solar radiation (horizontal surface), $\zeta$ is solar altitude angle, and $\theta$ is the zenith angle. Incident solar radiation reaching the ground is not simply proportional to the sine of solar altitude angle (or the cosine of zenith angle), but is influenced by surface albedo, clouds, particle 
scattering and absorption in the calculation of actual solar radiation. The studies of Liao et al. [1] on the scattering sulfate and strongly absorbing soot aerosols show that their respective radiative forcings at the top of the atmospheric vary as the cosine of the zenith angle changes. When $\cos \theta$ equals 0.35 , negative clear-sky radiative forcing by sulfate peaks, then decreases gradually as $\cos \theta$ increases. The studies of Nemesure et al. [4], Pilinis et al. [5] and Russell et al. [6] show similar trends. Positive clear-sky radiative forcing by soot at the top of the atmosphere increases as $\sin \zeta$ increases, and peaks when $\sin \zeta$ equals 1 , while clouds may enhance the impact of solar altitude angle. Because dust aerosols can both absorb and scatter solar radiation, and dust particles vary in absorption and scattering ability in different places [7], the impacts of solar altitude angle on dust direct radiative forcing at the top of the atmosphere and the atmospheric radiative heating rates are even more complicated than sulfate and soot, as demonstrated by studies on Saharan dusts. These studies [3] show that solar altitude angle has a greater impact on dust radiative temperature change than surface albedo. Comparing the curves for dust-induced radiative temperature changes where $\sin \zeta=0.8$ and 0.25 , the Saharan dusts can heat the atmosphere whatever the surface conditions when $\sin \zeta=$ 0.8 , while under $\sin \zeta=0.25$, the Saharan dusts can either heat or cool the atmosphere.

Although previous studies note the impact of changing solar altitude angle on aerosols, including the dust aerosol radiative effect, they have usually adopted a certain maximum or minimum value of $\sin \zeta$ or $\cos \theta$, or discussed impacts of daily solar altitude angle changes on radiative forcing at the top of the atmosphere or radiative heating (cooling) along the supposed vertical aerosol profile. Up to now few studies have addressed the day by day influence of $\sin \zeta$ changes on dust direct radiative effects. In East Asia, dust storms occur from winter to summer. During the highfrequency storm period from early to late spring, $\sin \zeta$ varies widely day by day. For dust storms occurring on different dates, the direct radiative effect may have different impacts on the earth-atmosphere system based on the specific $\sin \zeta$ at that time. However, this impact has not been addressed and quantified. Based on typical dust storms that occurred in 2001 over the East Asia-North Pacific region, and actual changes in both spring and annual $\sin \zeta$, this study analyzes the impacts of changing $\sin \zeta$ on dust direct radiative effects.

\section{Data and model description}

Data used in this analysis were dust concentration, complex refractive indices of dust aerosols, meteorological data such as temperature, atmospheric pressure and humidity, and surface conditions such as land cover type and desert distribution. Dust concentrations come from the Northern
Aerosol Regional Climate Model (NARCM) sand transport component, produced by the Canadian National Environment Agency [9,10], for spring 2001. The model uses Northern Hemisphere stereographic projection with $100 \mathrm{~km}$ $\times 100 \mathrm{~km}$ resolution, its $30 \mathrm{~km}$ vertical coordinate is divided into 22 layers and accounts for mixed terrain, and has 20 min time steps. The model can forecast mass concentration of dust particles $\left(\mu \mathrm{g} \mathrm{m}^{-3}\right)$. The study region covers East Asia, the northern Pacific and the northwest of the Americas $\left(75^{\circ} \mathrm{E}-225^{\circ} \mathrm{W}, 16^{\circ}-70^{\circ} \mathrm{N}\right)$. The dust concentration data cover these same regions, and have been verified by the Environmental Monitoring Network of the China Environmental Protection Administration, Canadian Air Forecast Monitoring Network, Canadian Aerosol Monitoring Network, and 13 ground monitoring stations including Aksu, Dunhuang, Yulin, Changwu, Urumqi, Hohhot, Changchun, Beijing, Shanghai and Qingdao in China, Seoul in Korea, and Saturna Island and Cheek Peak in North America. We adopted the complex refractive indices of dust aerosols from the East Asia Dust Particles Optical Model, which was obtained by aerosol sampling and measurement around Qira Desert in western China. When we compared these data with the dust optical models for Sahara and Central Asia [7], we found that it differed significantly, with stronger scattering and comparatively weaker absorption, and more closely resembles the optical properties of dust particles from East Asia and China.

The meteorological data (temperature, air pressure and humidity) were from the National Center for Environmental Prediction and National Center for Atmospheric Research (NCEP/NCAR), with $1^{\circ} \times 1^{\circ}$ resolution (http://dss.ucar.edu/ pub/reanalysis/). Surface albedo represents the absorption and reflection of solar radiation by the ground according to surface conditions and land cover, and is an important factor affecting sign of radiative forcing. We used the NARCM land cover data which is used in the Biosphere-Atmosphere Transfer Scheme (BATS) [11] to represent albedo. The data have $1 \mathrm{~km} \times 1 \mathrm{~km}$ resolution, and over the Chinese mainland utilize the latest desert cover map showing desertification in China from 1980s to the beginning of this century. Based on previous study results $[9,10]$, we applied a weighted average to 15 data types at each $1 \mathrm{~km} \times 1 \mathrm{~km}$ grid point to calculate surface albedo (Table 1). We did not consider changes in surface albedo that occur with changing wavelength of solar incident radiation, but instead used average albedo for all wave bands.

The radiation transfer model used here adopted a K-distribution as the basic frame for radiation calculation. It has a clear physical interpretation and precise results compareed to traditional line-by-line integration, which is utilized commonly to calculate atmospheric transmittance. The model includes a suitable method for cloud and aerosol radiation parameterization, and reasonably simulates radiative forcing and climate effects of global sulfate and soot aerosols [14-16]. Wang et al. [17,18] coupled the dust radiation parameterization 
Table 1 Land cover types and associated albedo

\begin{tabular}{clc}
\hline Type & \multicolumn{1}{c}{ Land cover } & Albedo \\
\hline 1 & evergreen coniferous forest & 0.12 \\
2 & evergreen broad-leaf forest & 0.12 \\
3 & deciduous coniferous forest & 0.17 \\
4 & deciduous broad-leaf forest & 0.17 \\
5 & coniferous and broad leaf forests & 0.12 \\
6 & grassland & 0.21 \\
7 & cultivated land & 0.20 \\
8 & desert & 0.25 \\
9 & tundra and frozen ground & 0.24 \\
10 & shrubs and bare ground & 0.17 \\
11 & wetland and marsh & 0.14 \\
12 & ice cap & 0.67 \\
13 & interior drainage & 0.08 \\
14 & ocean & 0.08 \\
15 & cities & 0.20 \\
\hline
\end{tabular}

scheme including instant dust forecasting concentration and latest dust optical performance into the model, which rapalces the climate values of key radiative parameters including optical thickness, single scattering albedo and dust asymmetry factors with temporal and special changing values on-line calculated by the dust model. It successfully calculated the radiative forcing of aerosols in the East Asia-North Pacific region in northern spring 2001, and evaluated the impact of optical performance of east Asian dust particles on direct radiative forcing and radiative heating rates. On the basis of this prior research, we further assess the impact of seasonal and annual variation in $\sin \zeta$ on dust radiative forcing and earth-atmosphere radiative effect in the East Asia-North Pacific region.

\section{Seasonal and annual variations in solar altitude angle over the East Asia-North Pacific region}

Studies over recent decades on east Asian dust storms [19-23] illustrate that dust storms occur throughout the year in southern Mongolia, at the Chinese-Mongolian border, and in western and northern China. Winter and spring are the high seasons for regional and strong dust storms. Some local dust storms occur in summer. Therefore, the variation of $\sin \zeta$ between dust storms can be extremely large. To better understand variability of dust direct radiative effects caused by seasonal changes in $\sin \zeta$, we first calculated the seasonal and annual variation trends of $\sin \zeta$ in the region.

The solar altitude angle, $\zeta$, depends on local time and longitude and latitude. We adopted the following formula [24]:

$$
\sin \zeta=\sin \phi \sin \delta+\cos \phi \cos \zeta \cos t_{\text {local }}
$$

$$
\begin{gathered}
\delta=0.006918-0.399912 \cos \theta_{0} \\
+0.070257 \sin \theta_{0}-0.006758 \cos 2 \theta_{0} \\
+0.000907 \sin 2 \theta_{0}-0.002697 \cos 3 \theta_{0} \\
+0.001480 \sin 3 \theta_{0}, \\
\theta_{0}=\frac{2 \pi d}{365}, \\
t_{\text {loctm }}=\mathrm{GMT}+\mathrm{XLONG} / 15, \\
t_{\text {local }}=15\left(t_{\mathrm{loctm}}-12\right), \\
\text { deg rade }=\pi / 180 .
\end{gathered}
$$

Here, $\phi$ refers to the latitude, $d$ is the calendar date (January 1 is expressed as 0$)$, XLONG is the local longitude east from the Greenwich meridian, $t_{\text {loctm }}$ is local time, $t_{\text {local }}$ refers to the hour angle, and GMT is Greenwich Mean Time. The $\zeta$ is primarily the function of longitude, latitude and local time.

Because we aimed to evaluate changes in direct radiative effect by dust caused by varying $\sin \zeta$ on different dates, and the dust concentration from NARCM refers to the daily average value, in the following calculation and analysis the daily average $\sin \zeta$ refers to the arithmetic mean value of $\sin \zeta$ during the daytime of a calendar date, while the variation of $\sin \zeta$ means the variation of the daily averaged $\sin \zeta$. In this section we firstly calculate the daily average variation of $\sin \zeta$ along the latitude from the first day of spring (March 1) to the last day spring (May 31) and from December 22 to June 22 in the simulated region (Figure 1). The variation of $\sin \zeta$ and changes in calendar days is very significant at different latitudes. The variation of $\sin \zeta$ and changes of calendar days is very large at higher latitudes. From March 1 to May 31, sin $\zeta$ increases by $20 \%$ in low latitudes areas, $30 \%$ to $40 \%$ at middle latitudes, and by $60 \%$ at high latitude areas. From December 22 to June 22, the variation of $\sin \zeta$ is even stronger, with $30 \%-40 \%$ increase at low latitudes, $60 \%$ at the middle latitudes area and $80 \%$ at high latitudes. This shows that the seasonal and annual variation of the daily averaged $\sin \zeta$ is very large. So, what differences will be made on the earth-atmosphere system radiation effect by dusts of the dust storms occurring in the early spring (or winter), or late spring (or early summer)? We further investigate this in the next section.

\section{Effect of solar altitude angle on clear-sky dust radiative forcing}

Generally, instant clear-sky aerosol radiative forcing is defined as the difference in radiant flux between the air with aerosols and clear air. The calculation of the radiative force of clear sky at the top of the atmosphere $\left(\Delta F_{\mathrm{TOA}}\right)$ and at the 


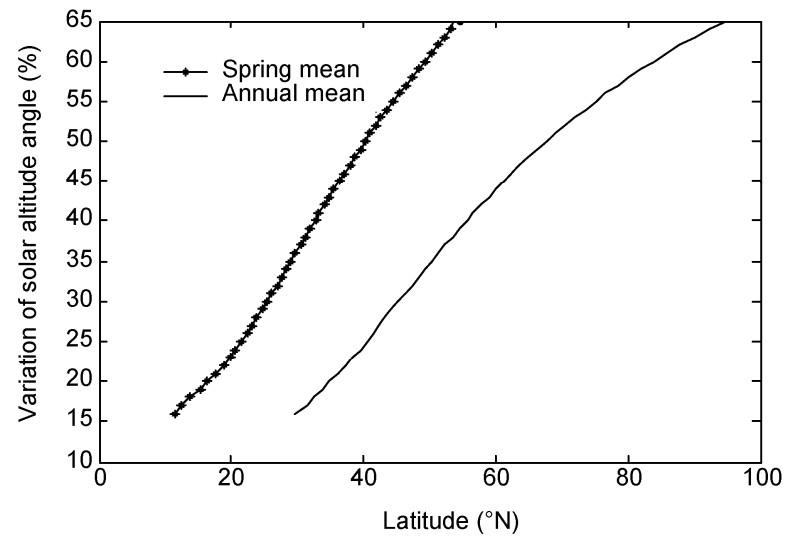

Figure 1 Variation of averaged $\sin \xi$ along latitude (\%).

ground $\left(\Delta F_{\mathrm{SFC}}\right)$ is as follows [3]:

$$
\begin{gathered}
\Delta F_{\mathrm{TOA}}=-\left(F_{\text {Solar }+ \text { IR }}\left(\uparrow_{\text {TOA,dusty }}\right)-F_{\text {Solar }+ \text { IR }}\left(\uparrow_{\text {TOA,clear }}\right)\right) . \\
\Delta F_{\mathrm{SFC}}=F_{\text {Solar }+ \text { IR }}\left(\downarrow_{\text {SFC, dusty }}\right)-F_{\text {Solar }+ \text { IR }}\left(\downarrow_{\text {SFC,clear }}\right) .
\end{gathered}
$$

Based on three typical dust storms that occurred in the East Asia-North Pacific region on March 2-6 (dust storm 1), April 6-15 (dust storm 2) and April 29-May 5 (dust storm 3) in 2001, Gong et al. [9,10] performed simulations, analyzed and verified the distribution characteristics of dust aerosol mass concentration in their period studies. We utilized the mass concentration of dust aerosols simulated by Gong et al. as the input data of the radiation transfer model, and calculated the average clear sky radiative forcing at the top of the atmosphere (Figure 2) of the three dust storms, and compared the three. For dust storm 1 which occurred in the early spring, the dusts lead to negative radiative forcing at the top of atmosphere over both land and sea within the whole simulated region, which illustrated that the dust layer decreased the temperature of the whole earth-atmosphere system. Over the boundary between China and Mongolia and the desert in northern Inner Mongolia, where there is high surface albedo, dusts had lower negative radiative forcing, which demonstrated that dusts had weaker impact on decreasing the temperature of the earth-atmosphere system. However, over Northeast China and China seas, with a lower surface albedo, dusts have larger negative radiative forcing, showing a greater impact of decreasing the temperature of the earth-atmosphere system.

Dust storm 2 occurred in the middle of spring. By comparing with the clear sky radiative forcing at the top of the atmosphere of dust storm 1, dust storm 2, which was stronger than dust storm 1 , had a greater atmospheric loading and optical thickness [17]. However, the negative radiative forcing at the top of the atmosphere over the Chinese mainland was smaller than that of dust storm 1 , and a small area over the desert in central Inner Mongolia at $40^{\circ} \mathrm{N}$, $105^{\circ} \mathrm{E}$ experienced positive radiative forcing. This shows that dusts increased slightly the temperature of the earth- atmosphere system in this region. In regard to oceans and similar to dust storm 1, dust storm 2 generated negative radiative forcing, and decreased the temperature of the earth-atmosphere system.

Dust storm 3 occurred in late spring, and showed opposite characteristics to dust storm 1. It generated a large area of positive radiative forcing over southern Mongolia, the boundary between China and Mongolia, and the desert in central Inner Mongolia. The radiative forcing had a value of $5 \mathrm{~W} \mathrm{~m}^{-2}$ at the center, meaning that the dusts significantly heated the earth-atmosphere system. However, dust storm 3 still decreased the temperature at sea. The comparative analysis of dust storms 1, 2 and 3 shows for dust storms occurring in early spring over the desert, the dust layer will significantly cool the earth-atmosphere system. For dust storms in the middle of spring, the dust layer may increase or decrease temperature slightly. Dust storms in late spring will heat the earth-atmosphere system. Dust layers over the oceans will generally cool the earth-atmosphere system, but that impact becomes weaker from early to late spring.

Because the clear-sky radiative forcing at the top of the atmosphere is also affected by the height of the dust layer, the optical thickness of dust and the surface conditions, the above phenomenon cannot fully demonstrate that the variation of $\sin \zeta$ is the cause of the sign change of the radiative forcing at the top of the atmosphere over the desert during the three dust storms. To further confirm its influence, based on the calculations and analysis of the previous section, the average $\sin \zeta$ varies from $30 \%$ to $40 \%$ in spring and even more in winter at the middle-latitudes, so here we present the following sensitivity test. To calculate the clear sky radiative forcing at the top of the atmosphere when the dust storm 2 occurred, we assumed that the variation of $\sin \zeta$ in this area was within $30 \%$, and adopted the $\sin \zeta$ as the actual calculation value, with $30 \%$ plus and $30 \%$ reduce on dust storm 2. Other items remain unchanged in the model. Comparing the radiative forcings at the top of the atmosphere among the three situations (Figure 3), the increase in $\sin \zeta$ can be seen to lead to the increase in positive radiative forcing and the decrease in negative forcing over both land and sea. In regard to central Inner Mongolia and the Loess Plateau, which have higher surface albedo and dust concentration, the $\sin \zeta$ plays a more important role, and its increase will change directly the negative radiative forcing into positive. For the Pacific and land with lower surface albedo, its increase may lead to decrease in negative radiative forcing, but its impact on dust radiation is much smaller than in the desert. Based on the above studies and analysis, we find that the higher-albedo surfaces such as desert and the Gobi will reflect much more solar radiation reaching the ground to the aerosol layer, which will further facilitate the multiple absorption and scattering. These areas are therefore more sensitive to variation in $\sin \zeta$. The domain-averaged negative radiative forcing of the simulated region (Table 2) will decrease along with increase of $\sin \zeta$ because of the positive 

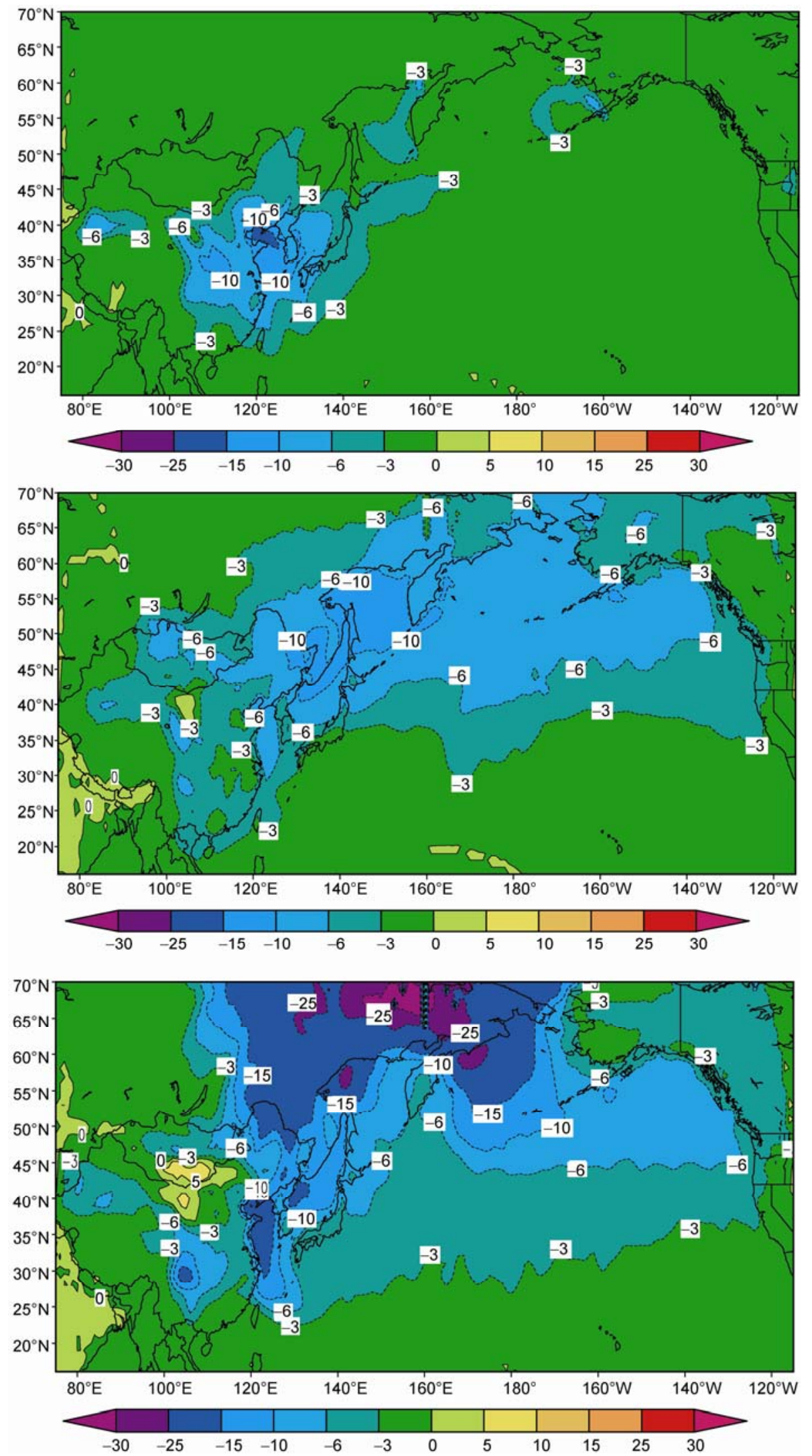

Figure 2 Clear-sky radiative forcing at the top of the atmosphere of dust storm 1 (top), dust storm 2 (middle) and dust storm 3 (bottom). 

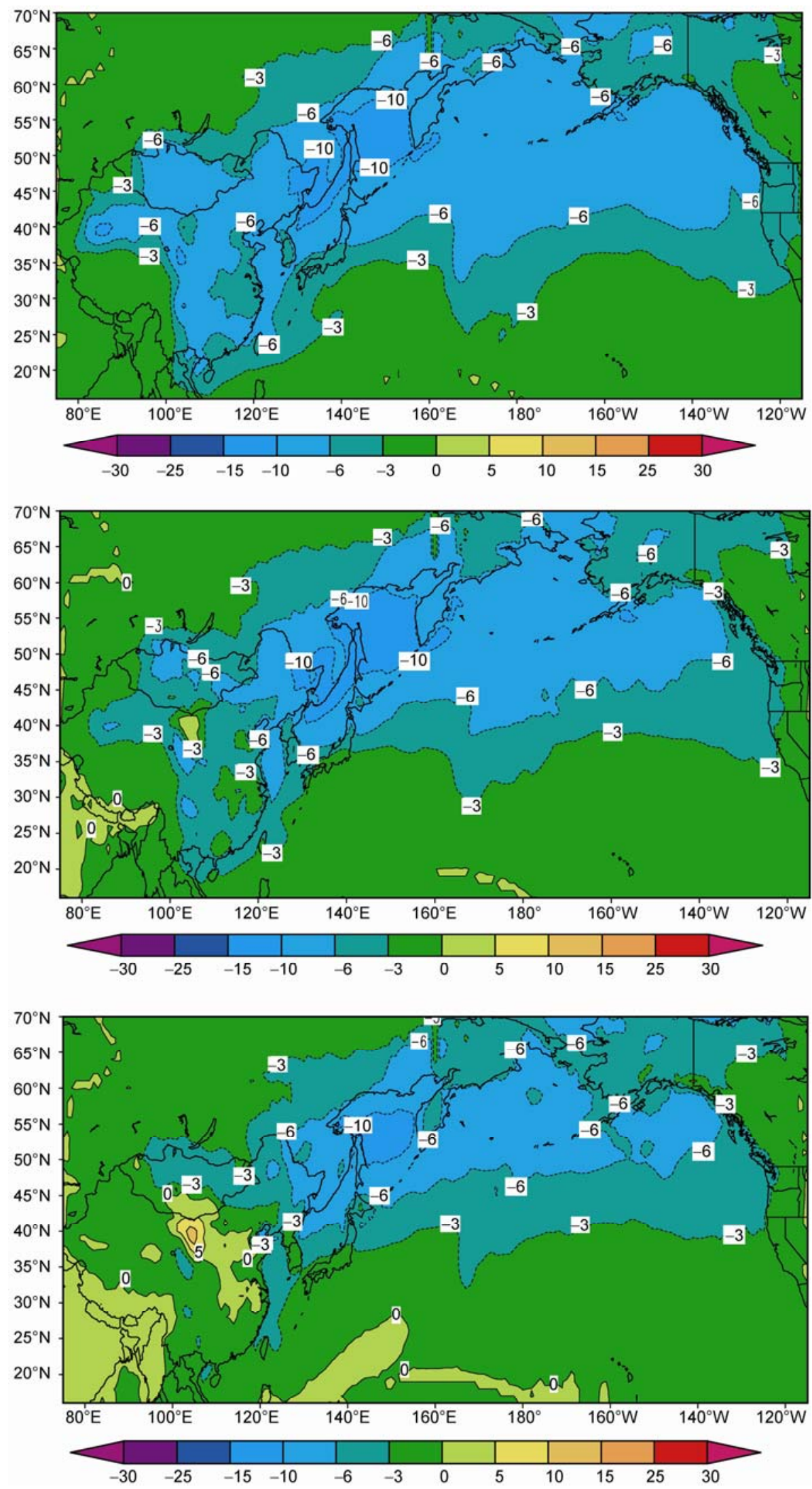

Figure 3 Clear-sky radiative forcing at the top of the atmosphere of $\sin \zeta$ decreased by $30 \%$ (top), ordinary (middle) and increased by $30 \%$ (bottom). 
Table 2 Shortwave radiative forcing at different $\sin \zeta$ for dust storm $3\left(\mathrm{~W} \mathrm{~m}^{-2}\right)$

\begin{tabular}{cccc}
\hline & \multicolumn{3}{c}{ Solar altitude angle } \\
\cline { 2 - 4 } & $0.7 \sin \zeta$ & $\sin \zeta$ & $1.3 \sin \zeta$ \\
\hline Top of the atmosphere & -4.971 & -4.310 & -3.348 \\
Ground & -9.081 & -10.020 & -10.276 \\
\hline
\end{tabular}

and negative values of different areas offsetting each other.

When $\sin \zeta$ increases, the incident solar radiation becomes stronger, then more radiation will be absorbed and scattered by the dust layer. Thus, a greater negative radiative forcing will be generated over land and sea, and therefore, the averaged ground negative forcing in this region increases with increase in $\sin \zeta$ (Table 2). This shows that the role of the dust layer in temperature decrease becomes greater in late spring compared to early spring.

According to the comparative studies on dust storms 1, 2 and 3 and the sensitivity test for $\sin \zeta$ of dust storm 2 , we can conclude the following. If a dust storm occurs close to winter, the $\zeta$ is lower, the clear sky radiative forcing at the top of the atmosphere of the dust is negative, and the dust layer will decrease the temperature of the earth-atmosphere system both over the desert and the sea. In contrast, if a dust storm occurs close to summer, the $\zeta$ is higher, the negative clear sky radiative forcing value of the dust at the top of the atmosphere is smaller over the oceans, and the dust layer plays a weaker role in decreasing temperature. Over the desert, the negative radiative forcing value at the top of the atmosphere will become positive as the $\sin \zeta$ increases, which will increase the temperature. That is to say, the dust storms in East Asia at different times and seasons have different impacts on the dynamic structure of atmosphere, and may even have opposite effects in desert regions. In summer, the dust layer has an impact on increasing the temperature of the earth-atmosphere system, while it significantly decreases air temperature in winter. Therefore the direct radiation effect of the dust layer is regarded as a cooling source in early spring (winter) and a warming source in late spring (summer) over East Asian deserts and nearby regions. This result also illustrates that the $\sin \zeta$ has a larger impact on the direct radiation effect of dust than does surface albedo. We note that $30 \%$ is the minimum value of the variation of daily averaged $\sin \zeta$ between early and late spring in middle latitude regions of East Asia, the actual value of which will be much more than $30 \%$ taking into account the difference between winter and summer and the variation of $\sin \zeta$ at noon. The resulting cooling in early spring (winter) and warming in late spring (summer) by the dust layer would be more significant than the calculated result presented here.

\section{The impact of solar altitude angle on cloudy sky radiative forcing}

In the previous section, we discussed the impact of $\sin \zeta$ on clear sky. However, clouds often coexist with aerosols. In this section we will discuss the impact of $\sin \zeta$ on cloudy sky radiative forcing, taking dust storm 2 as an example and comparing its similarities and differences with those of the clear sky condition.

\subsection{Clouds diagnostics scheme}

The impact of clouds on aerosols' radiative effect is very complicated, because it relates to a series of parameters including cloud height, thickness and their relative height to the aerosols, surface albedo, $\sin \zeta$ and the aerosols' optical characteristics. Different studies obtained different or even opposite result of the impacts of clouds on the radiative forcing of aerosols [1,3,25-29]. Wang et al. provided detailed discussion on the impact of clouds on dust radiative forcing and radiative temperature changes over East Asia in spring 2001 , and we refer the reader to their work. This section focuses on the impact of $\sin \zeta$ on cloudy sky radiative forcing, using dust storm 2 as an example. We adopt the same cloud diagnostics solution as refs. [7,17,29]. The cloud cover at any given layer of the model is calculated as

$$
A=\left(1.0-A_{\text {conv }}\right) A_{\mathrm{c}}+A_{\text {conv }},
$$

where $A$ is smaller than 0.999 . Here, $A_{\text {conv }}$ is the amount of convective clouds in the vertical layer, and $A_{\mathrm{c}}$ is the amount of medium and high clouds. The amount of convective clouds is calculated as

$$
A_{\text {conv }}=1.0-\left(1.0-\bar{A}_{\text {conv }}\right)^{1 / N} .
$$

Here, $N$ refers to the vertical layer of this model and $\bar{A}_{\text {conv }}$ is the total amount of convective clouds, which is calculated as

$$
\bar{A}_{\text {conv }}=0.035 \ln \left(1.0+\bar{M}_{\mathrm{c}}\right),
$$

where $\bar{M}_{\mathrm{c}}$ is the flux of average convective clouds on the vertical layer, which is calculated as

$$
\bar{M}_{\mathrm{c}}=\int_{p_{\mathrm{T}}}^{P_{\mathrm{S}}} m_{\mathrm{c}}(p) \mathrm{d} p / \int_{P_{\mathrm{T}}}^{P_{\mathrm{S}}} \mathrm{d} p,
$$

$m_{\mathrm{c}}(p)$ is the variance ratio of air pressure in units of $\mathrm{hPa} \mathrm{d}^{-1}$, and $P_{\mathrm{S}}$ and $P_{\mathrm{T}}$ refer to the air pressure at the bottom and top of the model, respectively. Medium and high clouds are calculated as

$$
\begin{gathered}
A_{\mathrm{c}}=\left[\max \left(0, \frac{R H-R H_{\text {lim }}}{1-R H_{\text {lim }}}\right)\right]^{2}, \\
R H_{\text {lim }}=0.999-\left(1-R H_{\text {mid }}^{\text {high }}\right)\left[1-\frac{N^{2}}{3.5 \times 10^{-4}}\right], \\
N^{2}=-\frac{g^{2} \rho}{\theta} \frac{\partial \theta}{\partial p},
\end{gathered}
$$

where $R H$ refers to the relative humidity from NCEP data, 
$R H_{\text {mid }}^{\text {high }}$ is the relative appropriate threshold value of medium or high clouds, confirmed by previous analyses. In this study, we confirm $R H_{\text {mid }}$ as 0.78 , and $R H^{\text {high }}$ as 0.65 . The cloud water path of this model is calculated as

$$
C W P(k)=\int_{z_{k}}^{z_{k+1}} A \cdot \exp \left(-z / h_{l}\right) \mathrm{d} z
$$

where $h_{1}=700 \times \ln \left(1+\int_{p_{t}}^{P_{s}} q \frac{\mathrm{d} p}{g}\right), Z_{k}$ and $Z_{k+1}$ refer to the height of the $k$ and $k+1$ layers of this model. $A$ is confirmed as $0.05 \mathrm{gm}^{-3}$, based on the data from SSM/I and GRODY [30].

Finally, the model calculates the amount of low clouds (Ground-700 hPa), medium clouds (700-400 hPa) and high clouds (above $400 \mathrm{hPa}$ ) by the free overlapping diagnosis. Based on the global average cloud types and thicknesses [31], the clouds are divided into five types (see Table 3) in the radiation transfer model. Low clouds include the continental stratus, marine stratus and cumulus clouds. Medium clouds are represented by alto stratus. The bottom of the cumulonimbus may be very low, or even similar to the height of low clouds, but the clouds top of the cumulonimbus may reach the height of the high clouds, and is the representative of high clouds. The radiation transfer model confirms the extinction coefficient, single scattering albedo and asymmetry factor for the five cloud types.

\subsection{The impact of variation in solar altitude angle on cloudy sky radiative forcing}

Current studies generally define cloudy sky radiative forcing as the difference in radiant flux between the atmosphere with clouds and aerosols and atmosphere with clouds only. The cloudy sky radiative forcing of dust at the top of the atmosphere and at the ground is calculated as follows [3]:

$$
\begin{aligned}
\Delta F_{\mathrm{TOA}}= & -\left(F_{\mathrm{Solar}+\mathrm{IR}}\left(\uparrow_{\mathrm{TOA}}, \text { dusty }+ \text { cloudy }\right)\right. \\
& \left.-F_{\text {Solar }+\mathrm{IR}}\left(\uparrow_{\mathrm{TOA}}, \text { cloudy }\right)\right), \\
\Delta F_{\mathrm{SFC}}= & F_{\text {Solar }+\mathrm{IR}}\left(\downarrow_{\mathrm{SFC}}, \text { dusty }+ \text { cloudy }\right) \\
& -F_{\text {Solar }+\mathrm{IR}}\left(\downarrow_{\mathrm{SFC}}, \text { cloudy }\right) .
\end{aligned}
$$

Table 3 Types of clouds

\begin{tabular}{lcc}
\hline \multicolumn{2}{c}{ Types of clouds } & Height of clouds \\
\hline Low clouds & marine stratus & over land, ground-900 hPa \\
& over sea, sea level-900 hPa \\
\hline Medium clouds & alto stratus & $900-700 \mathrm{hPa}$ \\
\hline High clouds & cumulonimbus & $700-400 \mathrm{hPa}$ \\
\hline
\end{tabular}

This section adopts the same sensitivity test as the clear sky radiative forcing. We adopted the $\sin \zeta$ as the actual calculation value, $30 \%$ plus and $30 \%$ reduce, to calculate the cloudy sky radiative forcing of dust storm 2. Comparing the three situations (Figure 4), the $\sin \zeta$ plays a similar role in the cloudy sky radiative forcing to that of clear sky. When $\sin \zeta$ increases, positive radiative forcing increases and the negative decreases or even becomes positive over land, while negative radiative forcing decreases over the oceans, and some negative regions become positive under the combined action of clouds and $\sin \zeta$. Unlike the clear sky radiative forcing, when the solar altitude angle is $30 \%$ plus on the actual value, the sign of radiative forcing at the top of the atmosphere over eastern China is the same as atmosphere over the desert in being slightly positive. This indicates that the dust layer slightly heats the earth-atmosphere system. According to the averaged radiative forcing of the simulation region, when the $\sin \zeta$ increases by $30 \%$, the cloudy sky radiative forcing at the top of the atmosphere decreases by $30 \%$ while the clear sky only drops by $22 \%$. This illustrates that the radiative forcing at the top of the atmosphere changes more with variation in $\sin \zeta$ when clouds are present. In other words, the occurrence of clouds enhances the impact of $\sin \zeta$ on the shortwave radiative forcing at the top of the atmosphere. This is because the low clouds are often located under the dust layer. This can increase the surface albedo and reflects more solar radiation back to the atmosphere. Therefore, when $\sin \zeta$ increases, more solar radiation will be reflected, so the dust cloudy shortwave radiative forcing at the top of the atmosphere changes more compared to that of clear sky. This result shows that the occurrence of low clouds may enhance the impact of $\sin \zeta$ on dust aerosol radiative forcing, bringing forward the time of when cooling changes to heating of the earth-at- mosphere system over the desert and even enabling dust direct radiative effects over east Asian land areas where low clouds occur widely. This is similar to dust direct radiative effects over desert, which act as a cooling source in early spring (winter) and a warming source in late spring (summer).

\section{Discussion}

To analyze the impact of solar altitude angle $(\sin \zeta)$ changing day-by-day on direct radiative effects during dust storms over East Asia and the North Pacific, we analyzed three typical dust storms, which occurred in the beginning, middle and end of spring 2001 in East Asia. We calculated the average clear sky radiative forcing occurring at the top of the atmosphere by the three dust storms. We found that from the beginning to the end of spring, the negative clear sky radiative forcing of the dust at the top of the atmosphere over desert in East Asia gradually turned positive, which 

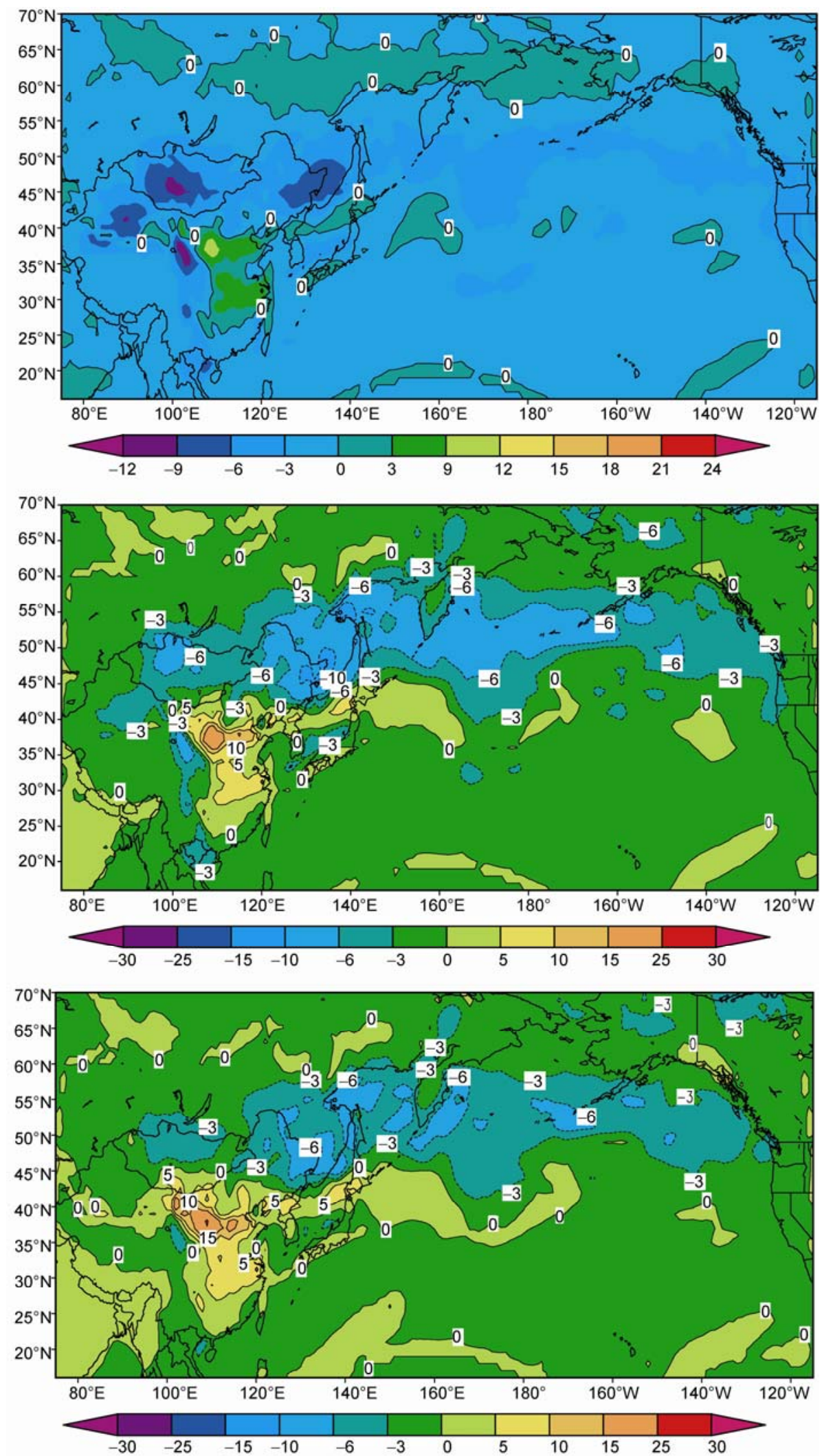

Figure 4 Dust cloudy sky radiative forcing at the top of the atmosphere of $\sin \zeta$ decreased by $30 \%$ (top), ordinary (middle) and increased by $30 \%$ (bothom). 
means that the dust changed from cooling to heating the earth-atmosphere system. To further confirm the impact of $\sin \zeta$ on this trend, we first calculated the average $\sin \zeta$ along this latitude for all of spring and its daily change from winter to summer, and designed a sensitivity test according to the variation range. We chose one dust storm and adopted the $\sin \zeta$ as increased and decreased by $30 \%$ respectively based on its calculation value, to calculate the clear sky and cloudy sky radiative forcing. By comparing the results of the radiative forcing at the top of the atmosphere of the three dust storms and the sensitivity test, we conclude the following.

In the East Asia-North Pacific region, from the early spring (winter) to the late spring (summer), the increase in $\sin \zeta$ leads to increase in positive clear sky radiative forcing at the top of the atmosphere and decrease of the negative radiative forcing caused by the dust storm. Over desert with high surface albedo, the increase in $\sin \zeta$ may lead negative radiative forcing at the top of the atmosphere to change to positive values, while the radiation alters from cooling the earth-atmosphere system to heating. This illustrates that the dust storm occurred in winter or early spring will cool the earth-atmosphere system over the desert, while the storm in late spring or summer will increase the earth-atmosphere temperature. Over oceans with lower surface albedo, the increase in earth-atmosphere from the early spring (winter) to late spring (summer) may lead to a decrease in the negative clear sky radiative forcing at the top of the atmosphere. This shows that the dust storms occurring in winter or early spring will strongly cool the ocean-atmosphere system, while those occurring in late spring or summer will only slightly decrease the temperature. For the dust storms closer to late spring or summer, more of the positive and negative radiative forcing over the land and oceans will offset each other and the negative radiative forcing at the top of the atmosphere of this region is smaller. The impact of the $\sin \zeta$ on the cloudy sky radiative forcing is similar to its impact on clear sky, except that the existence of low clouds enhances the impact of $\sin \zeta$ on the radiative forcing at the top of the atmosphere. On the one hand, not only in desert areas, but also over the land of east China and even part of the ocean, the increase of $\sin \zeta$ from the early spring (or winter) to late spring (summer) will change the cloudy sky radiative forcing from negative to the positive, leading to change from cooling to heating impacts on the earth-atmosphere system. On the other hand, low clouds bring forward the time of cooling changing to heating, by dusts according to the amount and height of clouds. The above analyses illustrate that dust storms with the same intensity over the East Asia desert and nearby regions may have different or even opposite direct radiative effects on the earth-atmosphere system including the thermal and dynamic structures of the earth-atmosphere system because of the different seasons and times of dust storm occurrence. The dust layer may be regarded as a cold source in early spring (winter) and a warming source in late spring (summer) over East Asian deserts and nearby regions.

This work was supported by the National High-Tech Research \& Development Program of China (2008BAC40B02), the Key Project of the Basic Research and Work Funding of Chinese Academy of Meteorological Sciences (2009z001), the National Department Public Benefit Research Foundation (GYHY200706036) and the National Natural Science Foundation of China (40775063).

1 Liao H, Seinfeld J H. Effects of clouds on direct aerosol radiative forcing of climate. J Geophy Res, 1998, 103: 3781-3788

2 Fouquart Y, Bronnel B, Brognig D, et al. Observation of Saharan aerosols: Results of ECLATS field experiment pat II-broadland radiative characteristics of the aerosols and vertical radiative flux divergences. J Clim Appl Meteor, 1987, 26:25-38

3 Qiujiano A L, Sokolik I N, Toon O B. Radiative heating rates and direct radiative forcing by mineral dust in cloudy atmospheric conditions. J Geophy Res, 105: 12207-12219

4 Nemesure S, Wagener R, Schwartz S E. Direct shortwave forcing of climate by anthropogenic sulfate aerosol: Sensitivity to particle size, composition, and relative humidity. J Geophys Res, 1995, 100: 2610526116

5 Pilinis C, Pandis S N, Seinfeld J H. Sensitivity of direct climate forcing by atmospheric aerosols to aerosol size and composition. J Geophys Res, 1995, 100:18739-18754

6 Russell P B, Kinne S A, Bergstrom R W. Aerosol climate effects: Local radiative forcing and column closure experiments. J Geophys Res, 1997, 102: 9347-9470

7 Wang H, Shi G Y, Li W, et al. The impacts of optical properties on radiative forcing due to dust aerosol. Adv Atmos Sci, 2006, 23: 431441

8 Redemann J, Turco R P, Liou K N, et al. Case studies of the vertical structure of the direct shortwave aerosol radiative forcing during TARFOX. J Geophys Res, 2000, 105: 9971-9980

9 Gong S L, Barrie L A, Blanchet J P, et al. Canadian Aerosol Module: A size-segregated simulation of atmospheric aerosol processes for climate and air quality models:1 Module development. J Geophys Res, 2003, 108, doi:10.1029/2001JD002002

10 Gong S L, Zhang X Y, Zhao T L, et al. Characterization of soil dust aerosol in China and its transport and distribution during 2001 ACE-Asia:2. Model simulation and validation. J Geophys Res, 2003, 108, doi:10.1029/2002JD002633

11 Dickinson R E, Henderson-Sellers A, Kennedy P J, et al. Biosphere-atmosphere transfer scheme (BATS) for the NCAR community climate model Boulder Colo, 1986

12 Wang B, The Radiation Transfer Model in Climate Simulation (in Chinese). Dissertation for the Doctoral Degree. Beijing: Institute of Atmospheric Physics of Chinese Academy of Sciences, 1996

13 Shi G Y. On the K-distribution and correlated K-distribution models in the atmospheric radiation calculations (in Chinese). Atmos Sci, 1998, 22: 659-676

14 Zhang L H, Shi G Y. The Impact of relative humidity on the radiative property and radiative forcing of sulfate aerosol. Act Geol Sin, 2001, 15: 465-476

15 Wang X H, Shi G Y, Ma X Y. Radiative forcing and temperature response of the anthropogenic sulfate in convection layer of East Asia (in Chinese). Atmos Sci, 2002, 26: 33-42

16 Ma X Y, Guo Y F, Shi G Y, et al. Numerical simulation of global temperature change during the 20th century with the IAP/LASG GOALS Model. Adv Atmos Sci, 2004, 2: 77-85

17 Wang H, Shi G Y, Aoki T, et al. Radiative forcing due to dust aerosol over east Asia-north Pacific region during spring 2001. Chinese Sci Bull, 2004, 49: 2212-2219 
18 Wang H, Shi G Y, Wang B, et al. Radiative heating characteristics of aerosol over chinese desert on the dessert and North Pacific (in Chinese). Atmos Sci, 2007, 31: 515-526

19 Zhou Z J, Wang X W. Establishment and analysis on the sequence of strong dust storms in the east of the North West China. Act Geol Sin, 2002, 57: 437-442

20 Yang D B, Shang K Z, Wang S G. Dust Storms. Beijing: Meteorological Publishing House, 2003. 39-45

21 Yue H, Wang X W, Li Y Q, et al. Analysis and Study on the Cases of Strong Dust Storms in Gansu (1955-2002). Beijing: Meteorological Publishing House, 2003. 1-50

22 Zhang X Y, Gong S L. Dust Storms in Northeast Asia in Spring 2006. Beijing: Meteorological Publishing House, 2006. 1-118

23 Chen Y, Chen D H, Wang H, et al. Numerical simulation test on dust storms in Hexi corridor in summer (in Chinese). Met Sci Tech, 2007, 35: 393-399

24 Paltridge G W, Platt C M R. Radiative Processes in Meteorology and Climatology. Amsterdam: Elsevier Press, 1976. 1-318

25 Carlson T N, Benjamin S. Radiative heating rates for Sahara dust. J
Atmos Sci, 1980, 37: 193-197

26 Woolword S. Modeling the atmospheric life cycle and radiative impact of mineral dust in the Hadley center climate model. J Geophys Res, 2001, 106: 18155-18166

27 Jacobson M Z. Global direct radiative forcing of multicomponent anthropogenic and natural aerosol. J Geophys Res, 2001, 106: 15511568

28 Myhre G, Stordal F. Global sensitivity experiments of the radiative forcing due to mineral aerosols. J Geophy Res, 2001, 106: 18193-18204

29 Wang H. Radiative forcing of dust aerosols in East Asia-North Pacific and its impact on the thermal structure of atmosphere (in Chinese). Dissertation for the Doctoral Degree. Beijing: Institute of Atmospheric Physics of Chinese Academy of Sciences, 2004. 1-143

30 Chen S H, Vandenberghe F, Petty G, et al. Application of SSM/I satellite data to a hurricane simulation. Quart J Rey Meteor Soc, 2004, 130: $801-825$

31 Liao X, Rind D, Rossow W B. Comparison between SAGE II and ISCCP high-level clouds, Part II: Locating cloud tops. J Geophys Res, 1995, 100: 1137-1147

Open Access This article is distributed under the terms of the Creative Commons Attribution License which permits any use, distribution, and reproduction in any medium, provided the original author(s) and source are credited. 